\title{
MOOD DISORDER (MANIA) INDUCED BY SYSTEMIC LUPUS ERYTHEMATOSUS, SUCCESSFULLY TREATED WITH OXCARBAZEPINE - A CASE REPORT
}

Nayana Naik ${ }^{1}$, Yvonne Da Silva Pereira² ${ }^{2}$ Ashish Srivastava ${ }^{3}$

\section{HOW TO CITE THIS ARTICLE:}

Nayana Naik, Yvonne Da Silva Pereira, Ashish Srivastava. "Mood disorder (mania) induced by systemic lupus erythematosus, successfully treated with oxcarbazepine- A case report". Journal of Evolution of Medical and Dental Sciences 2013; Vol2, Issue 31, August 5; Page: 5759-5763.

\begin{abstract}
It is well known fact that Systemic lupus erythematosus (SLE) may present with various neuropsychiatric manifestation, hence there are many presentation such as psychosis, mood disorder, anxiety disorders, organic brain syndrome, seizures, cerebrovascular accidents, transverse myelitis, , acute confusion and cognitive dysfunction. The Psychiatric symptoms in SLE can be functionally independent Psychiatric disorder or may be due to drugs especially steroids used for SLE, or secondary to SLE due to brain involvement which is termed as neuropsychiatric systemic lupus erythematosus (NPSLE). Although the exact immunopathological mechanism for psychiatric presentation remains elusive, prompt exclusion of other factors contributing to the psychiatric symptoms coupled with effective assessment strategies and management with immunosuppression and psychopharmacotherapy are imperative. Psychiatrists and rheumatologists must work in close liaison to identify, treat and prognosticate patients with psychiatric syndromes in order to improve their quality of life, vocational aptitude and, ultimately, survival. We report a case of 30 year old lady with organic manic disorder induced by Systemic lupus erythematosus successfully treated with oxcarbazepine (OXC), an adjunct to immunosuppressive therapy. To our knowledge this is the first case of its kind being successfully treated with oxcarbazepine.
\end{abstract}

KEY WORDS: neuropsychiatric systemic lupus erythematosus, mood disorder, mania, steroids, oxcarbazepine, systemic lupus erythematosus.

INTRODUCTION: SLE is chronic, multisystem, inflammatory disorder of connective tissue whose course is punctuated by exacerbation and remissions. It characteristically affects joints, although any system can be involved.

It is predominantly a disease of young females with peak incidence occurring between 20 and 40 yrs with a female to male ratio of 10:1.1 The exact patho-aetiology of systemic lupus erythematosus (SLE) remains elusive. An extremely complicated and multifactorial interaction among various genetic and environmental factors is probably involved. Multiple genes contribute to disease susceptibility. The interaction of sex, hormonal milieu, and the hypothalamo-pituitaryadrenal axis modifies this susceptibility and the clinical expression of the disease. ${ }^{2}$

As per American college of rheumatology revised criteria (1982) for classification of Systemic lupus erythematosus (SLE). ${ }^{3}$ A person shall be said to have SLE if any 4 of the 11 criteria are present. They are malar rash, discoid rash, photosensitivity, oral ulcers, arthritis, serositis, renal disorder, neurological disorder, hematological disorder, immune disorder, and antinuclear antibody.

Neuropsychiatric manifestation of SLE (NPSLE) is one of the major and most damaging presentations. It comprises a wide range of neurological syndromes affecting the central, peripheral and autonomic nervous systems, as well as psychiatric syndromes. In view of the diverse clinical 
manifestation of NPSLE, the American College of Rheumatology research committee devised a nomenclature which gives case definitions for 19 neuropsychiatric syndromes in SLE. ${ }^{4}$ listed in Table 1

Table 1 Neuropsychiatric manifestation of SLE

\begin{tabular}{|l|l|}
\hline NPSLE associated with Central nervous system & NPSLE associated with peripheral nervous \\
Aseptic meningitis & system \\
Cerebrovascular disease & Acute inflammatory demyelinating \\
Demyelinating syndrome & polyradiculoneuropathy \\
Headache (including migraine & syndrome) \\
and benign intracranial hypertension) & Autonomic disorder \\
Movement disorder (chorea) Myelopathy & Mononeuropathy (single/multiplex) \\
Seizure disorders & Myasthenia gravis \\
Acute confusional state (<1\%) & Cranial neuropathy \\
Anxiety disorder & Plexopathy \\
Cognitive dysfunction (55-80\%) & Polyneuropathy \\
Mood disorder $(14-57 \%)$ & \\
Psychosis (0-8\%) &
\end{tabular}

Systemic SLE lupus erythematosus; NPSLE-Neuropsychiatric Systemic lupus erythematosus

CASE REPORT: M.S. 29 year old married female from low socioeconomic background reported to the hospital on 31/05/2013 with body aches, migratory arthritis, intermittent low grade fever, and muscle weakness for 4 months duration. She had 20 days history of disturbed sleep, irritability, abusive and aggressive behavior, persecutory ideas against family members and her husband. She was overactive, talking more than usual, with neglect of self care and was unmanageable at home. There was no history suggestive of seizures or delirium. No history of substance use was elicited.

There was no past history of psychiatric or neurological disorder. Her premorbid personality was well adjusted.

Family history: she was born of a full term normal delivery, second of four siblings. Her mother expired when she was 8 years old. Father remarried. Her childhood was emotionally traumatic. She 


\section{CASE REPORT}

had to drop out of school after $7^{\text {th }}$ grade. Married at 18 years with two children aged 10 years and 7 years. There was no family history of Psychiatric or neurological illness.

On mental status examination: Young adult female with dishevelled appearance, conscious, partially co operative, irritable, increased psychomotor activity, poor rapport, with fleeting attention. She had increased tone of speech, circumstantiality, and air of confidence and increased self-esteem. Her mood was anxious and affect was irritable. She denied any perceptual disturbance. She was oriented to time place and person and her memory was intact. Her intelligence was average. Personal judgment was impaired.

Physical examination: She had oral ulcers. Her temp was $100.1^{\circ} \mathrm{F}$ respiratory system, cardiovascular system examination was normal. Central nervous system did not reveal any focal deficit.

Laboratory investigations: $\mathrm{Hb}$ was $12.1 \mathrm{gms} \%$, Erythrocyte (RBC) count 4.13 million /cu mm, total leucocytes count were within normal limits. Her ESR was raised to $52 \mathrm{~mm} / 1 \mathrm{hr}$. Her serum electrolyte, creatinine and blood urea were within normal limits. Her urine microscopy showed 89/hpf epithelial cells and 4-6/hpf pus cells. 24 hour urine protein was $2.5 \mathrm{gm} / \mathrm{l}$ in $24 \mathrm{hrs}$. Her serum albumin was $4.4 \mathrm{gm} / \mathrm{dl}$, globulin $2.7 \mathrm{gm} / \mathrm{dl}$ and AG ratio was 1.6. Her rest of Liver function tests (LFT) were normal. 25 - Hydroxyvitamin D was $28.89 \mathrm{ng} / \mathrm{ml}$. Antistreptolysin titre was negative. And C reactive protein was $25 \mathrm{mg} / \mathrm{l}$, fasting blood sugar level as $110 \mathrm{mg} / \mathrm{dl}$, uric acid $3.2 \mathrm{mg} / \mathrm{dl}$ and TSH was $3.57 \mathrm{miu} / \mathrm{ml}$. Her antinuclear antibody factor (ANA) was positive with titre of 1:40 litre and Antidouble stranded DNA antibody (ANTI-dsDNA Antibody) of $>200 \mathrm{IU} / \mathrm{ml}$. (ref. range $<35: 00 \mathrm{IU} / \mathrm{ml}$ ) .Her brain computed tomography (CT) and electrocardiogram (ECG) did not reveal any abnormality.

The patient was diagnosed to have organic manic disorder as per ICD 10- F06.30.5 secondary to SLE. She was treated in consultation with physician, with $50 \mathrm{mg} /$ day of prednisolone and hydroxyquinolone $400 \mathrm{mg}$ /day, along with mood stabilizer, Oxcarbazepine [OXC] 600mg /day in divided doses. Patient mood symptoms showed response to OXC and SLE symptoms also responded to treatment, over a period of next 2-3 weeks.

At her last follow up on 12/07/2013 she was mildly cheerful and had mild pressure of speech, hence her OXC was increased to $900 \mathrm{mg} /$ day.

DISCUSSION: About 28-40\% of neuropsychiatric SLE findings arise before or around the time of diagnosis. ${ }^{6} \mathrm{~A}$ psychotic episode due to SLE is most likely to occur during an acute exacerbation of the illness while the steroid psychosis are most likely to occur shortly after steroids are instituted or dose is increased. This distinction is very important in making correct diagnosis as insufficient doses of steroid may in fact contribute to the appearance of psychiatric symptoms as a result of inadequate control of the disease.

According to Monov and Monova 2008.7 Common differential diagnosis that need to be considered are functional psychosis, delirium, steroid psychosis and other drug induced psychosis.

The above noted patient did not have any past history of psychiatric illness; neither there was family history of bipolar disorder nor Psychosis. Hence functional psychosis was ruled out. Patient presented with acute mania around the time of diagnosis of SLE. Prednisolone and hydroxyquinolone was started only after positive ANTI- dsDNA antibody and ANA test. Her brain 
computed tomography (CT) did not reveal any abnormality, but neuroimaging and laboratory findings could be normal due to diffuse brain disease in SLE which could be difficult to assess due to its sparse biological expression. ${ }^{8}$ Metabolic causes were ruled out as she had normal renal, liver and thyroid function test. Also her electrolytes were normal. Based on the findings patient fulfilled the criteria for NPSLE as per American college of Rheumatology definition. ${ }^{9}$

As per our knowledge till date there are few reported cases in literature organic psychosis and organic mood disorder being successfully treated with typical and atypical antipsychotics and mood stabilizer like Valproic acid and carbamazepine and lithium.

Standard prescribing guidelines for mood disorder recommend a combination of an antipsychotic with lithium or, alternatively, a combination of an antipsychotic with valproate or carbamazepine. However, in our case of SLE induced mania, administration of lithium was at least relatively contra-indicated because the kidney is the most commonly involved visceral organ in SLE. Although only approximately $50 \%$ of patients with SLE develop clinically evident renal disease, biopsy studies demonstrate some degree of renal involvement in most patients. ${ }^{10}$

Furthermore, typical antipsychotics are prone to cause severe extra pyramidal symptoms and atypical antipsychotics like olanzapine and risperidone are associated with metabolic syndrome and hyperprolactinaemia respectively. As fatty liver is common in SLE, valproate may not have been a good choice hence OXC was selected as monotherapy as it is comparatively safe and efficacious in the treatment of acute mania then Carbamazepine. ${ }^{11}$ Patients with SLE who's already compromised physical condition constitutes an absolute or relative contra-indication for the administration of standard treatments.

CONCLUSION: Psychosis in patient with SLE needs to be differentiated from functional psychosis, drug induced psychosis, metabolic cause and psychosis due to SLE, as treatment in each case will differ. Since these patient are more prone for side effects with standard treatment for acute mania, may be treated with mood stabilizer which has more safety profile. Successful treatment of SLE related mania with oxcarbazepine in our patient brings another therapeutic option to those patients with manic disorder, although controlled studies would be necessary to confirm this observation.

\section{REFERENCES:}

1. Lahita RG: Emerging concepts for sexual predilection in the disease systemic lupus erythematosus. Ann NY Acad Sci 1999, 876:64-69.

2. C C Mok, C S Lau: Pathogenesis of systemic lupus erythematosus. J Clin Pathol 2003; 56:481490 doi:10.1136/jcp.56.7.48

3. Tan EM, Cohen AS, Fries JF, Masi AT, McShane DJ, Rothfield NF, et al. The 1982 revised criteria for the classification of systemic lupus erythematosus. Arthritis Rheum 1982; 25:1271-7 and Hochberg MC. Updating the American College of Rheumatology revised criteria for the classification of systemic lupus erythematosus [letter]. Arthritis Rheum 1997; 40:1725.

4. ACR Ad Hoc Committee on Neuropsychiatric Lupus Nomenclature (1999) The American College of Rheumatology nomenclature and case definitions for neuropsychiatric lupus syndromes. Arthritis and Rheumatism; 42: 599-608. 
5. ICD- 10 Classification of mental and behavioral disorder-clinical description and diagnostic guidelines,world health organization Geneva.

6. Muscal E, Brey RL. Neurologic manifestations of systemic lupus erythematosus in children and adults. Neurol Clin. Feb 2010;28(1):61-73.

7. Monov S, Monova D.Classification criteria for neuropsychiatric systemic lupus erythematosus: Do they need a discussion? Hippokratia.2008 Apr-Jun;12(2):103-107

8. Munoz-Malaga A, Anglada JC, and Paez M. et al. Psychosis as the initial manifestation of systemic lupus erythematosus: the role of lupus band test and anti-ribosomal antibodies. Rev Neurol. 1999. 28:779-781.

9. American College of Rheumatology. Appendix A: case definitions for neuropsychiatric syndromes in systemic lupus erythematosus. Available from: http://www.rheumatology.org/publications/ar/1999/aprilappendix.asp. Accessed April 25, 2012.

10. Wallace D, Edmund D, eds. Dubois' Lupus Erythematosus. Philadelphia, Pa: Lippincott Williams \& Wilkins.; 2006.

11. Dietrich DE, Kropp Emrich HM. Oxcarbazepine in affective and schizoaffective disorders. Pharmacopsychiatry. 2001 Nov; 34(6):242-50.

\section{AUTHORS:}

1. Nayana Naik

2. Yvonne Da Silva Pereira

3. Ashish Srivastava

\section{PARTICULARS OF CONTRIBUTORS:}

1. Assistant Professor, Department of Psychiatry, Institute of Psychiatry and Human Behaviour [IPHB].

2. Professor and HOD, Department of Psychiatry, Institute of Psychiatry and Human Behaviour [IPHB].

3. Lecturer, Department of Psychiatry, Institute of Psychiatry and Human

\section{NAME ADRRESS EMAIL ID OF THE CORRESPONDING AUTHOR:}

Dr. Nayana Naik,

BF-41, "OM HARI" Housing society, Goa Housing Board, Alto Porvorim Bardez, Goa, Pin - 403521.

Email-nnaik2002@yahoo.co.in

Date of Submission: 20/07/2013.

Date of Peer Review: 20/07/2013.

Date of Acceptance: 26/07/2013.

Date of Publishing: 31/07/2013 